\title{
Solving the shugoshin puzzle
}

\author{
Juraj Gregan, Mario Spirek, and Cornelia Rumpf \\ Max F. Perutz Laboratories, University of Vienna, 1030 Vienna, Austria
}

\begin{abstract}
Shugoshin proteins form a complex with protein phosphatase 2A (PP2A) that protects centromeric cohesin from separase-mediated cleavage during yeast meiosis I. Recent work shows that this mechanism is conserved from yeast to mammals. Importantly, a model emerges that explains a long-standing puzzle, namely why the shugoshin-PP2A complex mediates protection of centromeric cohesin from separase cleavage specifically during meiosis I, but not during meiosis II or mitosis.
\end{abstract}

\section{Meiotic chromosome segregation}

Chromosome number reduction occurs during meiosis by two rounds of chromosome segregation. Whereas the second meiotic division is similar to mitosis, with sister centromeres segregating to opposite poles, homologous centromeres segregate during the first meiotic division. Three major features of meiotic chromosomes ensure the reductional nature of chromosome segregation during meiosis I. The first is the formation of physical links, chiasmata, between two homologous chromosomes. The second meiosis I-specific feature is the attachment of sister kinetochores to microtubules emanating from the same spindle pole (mono-orientation). Recent reports shed light on the third meiosis I-specific feature: the protection of centromeric cohesin from separase-mediated cleavage [1,2].

\section{Shugoshin-protein phosphatase $2 \mathrm{~A}$ protects centromeric cohesin from separase-mediated cleavage during meiosis I}

Segregation of homologous centromeres during meiosis I is triggered by separase-mediated cleavage of the cohesin a-kleisin subunit, Rec8, along chromosome arms, whereas centromeric cohesin is protected from separase cleavage. This retention of centromeric cohesion is essential for sister-centromere bi-orientation and proper segregation during meiosis II.

The protection of centromeric cohesion during meiosis I is mediated by conserved proteins called shugoshins (Japanese for 'guardian spirit') (reviewed in Ref. [3]).

The role of shugoshins in mouse meiosis was recently analyzed [1]. Both mouse shugoshin proteins, Sgo1 and Sgo2, are ubiquitously expressed in proliferating cells, and Sgo2 expression is higher in the testis and oocytes. RNAi-mediated Sgo2 depletion in mouse oocytes resulted in precocious sister chromatid separation, whereas only a modest defect was observed in Sgo1-depleted oocytes. These results indicate that in oocytes Sgo2 depletion abolishes sister centromere cohesion in anaphase I. Consistent with this notion, whereas Sgo2 depletion did not affect Rec8 localization at metaphase I, Rec8 was absent at

(C) 2008 Elsevier Ltd. All rights reserved.

Corresponding author: Gregan, J. (juraj.gregan@univie.ac.at).. 
metaphase II. Does mouse Sgo2 regulate separase-mediated cleavage of centromeric Rec8? Separase proteolytic activity is essential for Rec 8 removal from chromosome arms during meiosis I in mouse oocytes [4]. Therefore, it is likely that $\mathrm{Sgo} 2$ is required to protect centromeric cohesin from separase-mediated cleavage.

Given the caveats associated with the RNAi technique, it will be important to analyze meiosis in shugoshin knockout cells. Because both sgol and sgo2 knockouts prevent the birth of homozygous knockout mice [1], the construction of conditional knockout alleles will be essential for further progress.

Previous biochemical analyses revealed that shugoshin proteins form a complex with a specific form of protein phosphatase 2A, called PP2A- $\pi$ [5-7]. Yeast shugoshins recruit PP2A to centromeric regions, where it is required to prevent cohesin cleavage during meiosis I, possibly by dephosphorylating Rec8p [5,6]. In agreement with these observations from yeast meiosis, the analysis of mouse oocytes revealed that PP2A co-localizes with Sgo2 on metaphase I centromeres. Importantly, PP2A centromeric localization was abolished in Sgo2-depleted oocytes [1]. These data suggest that PP2A might be required to protect centromeric cohesin from separase-mediated cleavage during mouse meiosis. This idea is further supported by the previous observation that okadaic acid, an inhibitor of PP1 and PP2A, induces precocious sister chromatid separation during meiosis I in mouse oocytes [8].

\section{A model for shugoshin-PP2A-mediated centromeric cohesion protection}

Surprisingly, shugoshins, key meiotic regulators, are not expressed only during meiosis I. Shugoshin and PP2A are also present at centromeres during mitosis and meiosis II. However, despite the presence of shugoshin and PP2A at centromeres, centromeric cohesin is sensitive to separase cleavage during mitosis and meiosis II (reviewed in Ref. [9]). Why does the shugoshin-PP2A complex mediate protection of centromeric cohesin from separase-mediated cleavage specifically during meiosis I but not during meiosis II and mitosis?

Detailed microscopy analysis of mouse oocytes revealed that Sgo 2 co-localizes with Rec8 on metaphase I centromeres, but in prometaphase II, Sgo2 relocates from the sites of cohesin-mediated sister chromatid cohesion, towards the kinetochore [1]. If shugoshinPP2A must be in close proximity to cohesin to protect it from separase-mediated cleavage, Sgo2 relocation away from cohesin sites provides an elegant explanation for why shugoshin-PP2A can no longer protect centromeric cohesin during meiosis II. What induces shugoshin to relocate from the cohesin sites? One obvious difference between meiosis I and meiosis II chromosomes is the orientation of sister kinetochores. In meiosis II, bi-oriented sister kinetochores are subjected to tension exerted by the pulling forces of the spindle. This tension across sister kinetochores might force the shugoshin-PP2A complex to relocate from cohesin sites at the inner centromere domain towards the inner kinetochore (Figure 1) [1,2].

A similar tension-dependent redistribution of shugoshin proteins has been observed in mouse spermatocytes, as well as in NIH-3T3 and HeLa cells $[2,6,10,11]$. However, the relevance of $\mathrm{Sgo} 2$ redistribution for the protection of centromeric cohesion remained elusive until Watanabe and co-workers [1] showed that in HeLa cells, centromeric cohesin is preserved if cells enter anaphase in the absence of tension across sister centromeres. In this key experiment, an artificial situation was created in which anaphase ensued without spindle formation. HeLa cells treated with nocodazole, a microtubule-destabilizing drug, arrested at prometaphase because of activation of the spindle checkpoint. Subsequently, Mad2 depletion was used to override the nocodazole arrest, and the cells entered anaphase in the absence of a functional spindle. A significant fraction of such 'tensionless anaphase' cells 
contained cohesin and shugoshin at their centromeres [1]. A simple explanation for the retention of centromeric cohesin in the 'tensionless anaphase' cells is cohesin resistance to separase-mediated cleavage.

The model proposed by Watanabe and co-workers [1] and Suja and co-workers [2] predicts that the shugoshin-PP2A complex cannot protect centromeric cohesion during meiosis I if sister kinetochores are bi-oriented. Indeed, studies in mouse oocytes and fission yeast mutants demonstrate that bi-oriented sister kinetochores frequently segregate to opposite poles during meiosis I (e.g. Refs. [12,13]). However, it is not clear whether centromeric cohesin is cleaved by separase or whether spindle pulling forces simply break centromeric cohesin. Saccharomyces cerevisiae monopolin mutants contain sister kinetochores that are bi-oriented during meiosis I; nevertheless, centromeric cohesin remains intact and resists spindle pulling forces, thus preventing the segregation of sisters to opposite poles [14]. In addition, ectopic co-expression of Rec8p and Sgolp in Schizosaccharomycas pombe cells causes failure of sister chromatid segregation during mitosis, presumably because of resistance of centromeric Rec8p to separase-mediated cleavage [15]. However, both of these examples in which centromeric cohesion prevents segregation of bi-oriented sisters to opposite poles are artificial and might not reflect the wild-type situation.

\section{Concluding remarks}

In summary, Watanabe and co-workers [1] now provide an important piece of evidence suggesting that the shugoshin-protein phosphatase 2A (PP2A)-mediated protection of centromeric cohesin during meiosis I is conserved from yeast to mammals. Cytological observations showed that shugoshin proteins are displaced from the sites of cohesinmediated sister chromatid cohesion when kinetochore microtubules exert tension across sister centromeres. This finding suggests an elegant model that explains why the shugoshinPP2A complex protects centromeric cohesin from separase-dependent cleavage only during meiosis I, but not during meiosis II or mitosis [1,2].

Reversible protein phosphorylation is the major regulatory mechanism in the cell, and the protection of centromeric cohesion seems to be no exception. Recent studies raise many questions concerning the interplay between kinases and phosphatases in regulating sister centromere cohesion. To fully understand this process, it will be important to identify centromeric targets of PP2A and to determine whether (and how) PP2A activity is regulated at centromeres.

\section{Acknowledgments}

The authors thank J. Suja and Y. Watanabe for helpful discussions and M. Siomos for critically reading this manuscript. Work in our laboratory is supported by the Austrian Science Fund (P18955-B03 and F34-B03) to J.G.

\section{References}

1. Lee $\mathrm{J}$, et al. Unified mode of centromeric protection by shugoshin in mammalian oocytes and somatic cells. Nat. Cell Biol. 2008; 10:42-52. [PubMed: 18084284]

2. Gomez R, et al. Mammalian SGO2 appears at the inner centromere domain and redistributes depending on tension across centromeres during meiosis II and mitosis. EMBO Rep. 2007; 8:173180. [PubMed: 17205076]

3. Watanabe Y, Kitajima TS. Shugoshin protects cohesin complexes at centromeres. Philos. Trans. R. Soc. Lond. B Biol. Sci. 2005; 360:515-521. [PubMed: 15897177]

4. Kudo NR, et al. Resolution of chiasmata in oocytes requires separase-mediated proteolysis. Cell. 2006; 126:135-146. [PubMed: 16839882] 
5. Riedel CG, et al. Protein phosphatase $2 \mathrm{~A}$ protects centromeric sister chromatid cohesion during meiosis I. Nature. 2006; 441:53-61. [PubMed: 16541024]

6. Kitajima TS, et al. Shugoshin collaborates with protein phosphatase $2 \mathrm{~A}$ to protect cohesin. Nature. 2006; 441:46-52. [PubMed: 16541025]

7. Tang Z, et al. PP2A is required for centromeric localization of Sgol and proper chromosome segregation. Dev. Cell. 2006; 10:575-585. [PubMed: 16580887]

8. Mailhes JB, et al. Okadaic acid, an inhibitor of protein phosphatase 1 and $2 \mathrm{~A}$, induces premature separation of sister chromatids during meiosis I and aneuploidy in mouse oocytes in vitro. Chromosome Res. 2003; 11:619-631. [PubMed: 14516070]

9. Gregan J, et al. What makes centromeric cohesion resistant to separase cleavage during meiosis I but not during meiosis II? Cell Cycle. 2007; 7:151-153. [PubMed: 18256525]

10. McGuinness BE, et al. Shugoshin prevents dissociation of cohesin from centromeres during mitosis in vertebrate cells. PLoS Biol. 2005; 3:e86. [PubMed: 15737064]

11. Huang H, et al. Tripin/hSgo2 recruits MCAK to the inner centromere to correct defective kinetochore attachments. J. Cell Biol. 2007; 177:413-424. [PubMed: 17485487]

12. Kouznetsova A, et al. Bi-orientation of achiasmatic chromosomes in meiosis I oocytes contributes to aneuploidy in mice. Nat. Genet. 2007; 39:966-968. [PubMed: 17618286]

13. Hauf S, et al. Aurora controls sister kinetochore mono-orientation and homolog bi-orientation in meiosis-I. EMBO J. 2007; 26:4475-4486. [PubMed: 17932486]

14. Petronczki M, et al. Monopolar attachment of sister kinetochores at meiosis I requires casein kinase 1. Cell. 2006; 126:1049-1064. [PubMed: 16990132]

15. Kitajima TS, et al. The conserved kinetochore protein shugoshin protects centromeric cohesion during meiosis. Nature. 2004; 427:510-517. [PubMed: 14730319] 
(a) Meiosis I

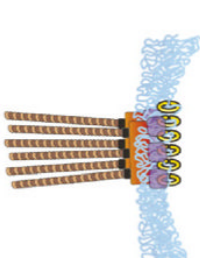

(b) Mitosis/Meiosis II low tension

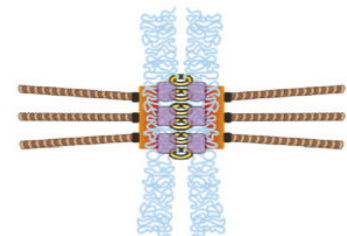

(c) Mitosis/Meiosis II high tension

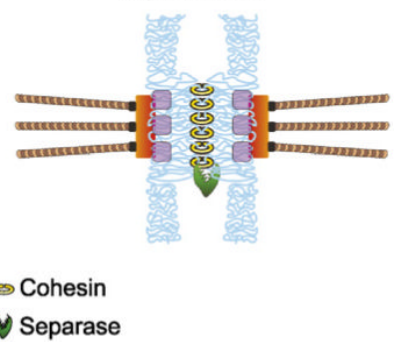

Figure 1.

A model of shugoshin-protein phosphatase 2A (PP2A)-mediated protection of centromeric cohesin. Co-localization of cohesin (yellow) with shugoshin-PP2A (purple) at centromeres prevents separase-mediated (green) cleavage of centromeric cohesin during meiosis I (a). Microtubules attached to bi-oriented sister kinetochores (orange) during mitosis and meiosis II (b) produce tension that promotes the relocation of shugoshin-PP2A from the centromeric cohesin sites (c). Sister centromeres and centromere-proximal regions of one homolog are shown. This model is based on the results from the Watanabe and Suja groups $[1,2]$. 